\title{
4DSTEM of Beam-sensitive Materials: Optimizing SNR and Improving Spatial Resolution
}

Karen Bustillo ${ }^{1}$, Steven Zeltmann ${ }^{2}$, Min Chen $^{2}$, Jennifer Donohue ${ }^{2}$, Alexander Mueller ${ }^{1,2}$, Colin Ophus ${ }^{3}$, Jim Ciston $^{3}$ and Andrew Minor ${ }^{2,3}$

${ }^{1}$ NCEM, Molecular Foundry, Lawrence Berkeley National Laboratory, Berkeley, California, United States, ${ }^{2}$ University of California-Berkeley, Berkeley, California, United States, ${ }^{3}$ Lawrence Berkeley National Lab, Berkeley, California, United States

Four-dimensional scanning transmission electron microscopy (4D-STEM) [1], or scanning nanobeam diffraction, can be used to produce maps of several material properties while also minimizing dose and damage in beam-sensitive materials. Many technologically interesting materials are challenging to study using TEM because they are both electron-beam-sensitive and weakly scattering to electrons. These materials are often comprised of low $\mathrm{Z}$ elements such as carbon, and are structurally inhomogeneous, containing regions of amorphous or medium-range order mixed with small, randomly-oriented crystallites of various quality. 4D-STEM is advantageous for such systems because it provides an efficient use of the illuminating electrons, capturing the entire distribution of electron scattering at every scan position, while also providing spatial resolution of a few to 10 nanometers. In practice, 4D-STEM of beam-sensitive materials is often a 'diffract and destroy' technique, so optimization of the signal-to-noise ratio (SNR) in the diffraction patterns is essential.

Here, we will discuss various approaches to minimizing electron beam damage while maximizing SNR during 4D-STEM experiments on beam-sensitive materials. Examples will include experimental data from soft materials including polymer and small molecule blends [2], peptides [3], and 2D nanosheets. The techniques discussed can also be applied to metal-organic and covalent-organic frameworks, beamsensitive perovskites, and battery solid electrolytes. In addition to using cryogenic temperature (liquid $\mathrm{N}_{2}$ ) to reduce damage from the electron beam, several other experimental approaches that improve SNR in the diffraction pattern will be presented: the use of small condenser apertures to achieve nearly-parallel STEM probes, shaping of the real space probe, and a comparison of different electron detector types.

Using small apertures in the condenser system to make the scanning probe more parallel improves the SNR in the diffraction pattern. In a typical high-resolution STEM image, the probe convergence angle is 10 s of mrad and consequently the diffracting electrons are spread over the pixels in the disk, which in weakly scattering systems reduces the counts per pixel to be near the noise floor. By using custom FIBmilled apertures of 2-20 $\mu \mathrm{m}$ and reducing the convergence angle to the sub-mrad level, we are able to detect diffraction that would otherwise not be possible in some polymer and small molecule systems. These smaller apertures also have the advantage of reducing the illumination dose rate beyond the minimum normally attainable from the spot size control. The disadvantage of a more parallel illumination is the increase in the size of the probe in real space, which then reduces the spatial resolution in the resulting maps and virtual images. The tradeoffs between SNR and real-space spatial resolution for various aperture sizes will be presented with experimental and simulated data for different 4D-STEM analyses, including strain mapping and radial distribution function calculation [4]. In addition, the shape and size of the real space probe can be changed from a focused Airy-disk profile to a defocused profile that distributes the electron intensity over a larger region for each scan position. Experimental data to optimize the probe shape and its consequence on SNR and spatial resolution will be presented similar to previously reported findings [5]. 
Four different camera types were used to acquire 4D-STEM data of beam-sensitive materials and will be discussed: a direct-electron counting detector (Gatan K3), a direct-electron integrating detector operating in in-situ mode (Gatan K2), a scintillator-CMOS detector (Thermo Fisher Scientific Ceta 2), and a scintillator-CCD detector (Gatan Orius). These detectors differ in frame rates, dynamic range, and noise characteristics, properties that have large effects on the SNR of 4D-STEM datasets.

Finally, measuring diffraction contrast in a beam-sensitive sample requires that the sample remains intact for long enough to acquire diffraction patterns with sufficient SNR. In parallel beam illumination, one can measure the decay time of the diffraction using selected area diffraction. Methods for ascertaining the decay time using scanning nanobeam diffraction and quantifying the dose for this mode will be presented [6].
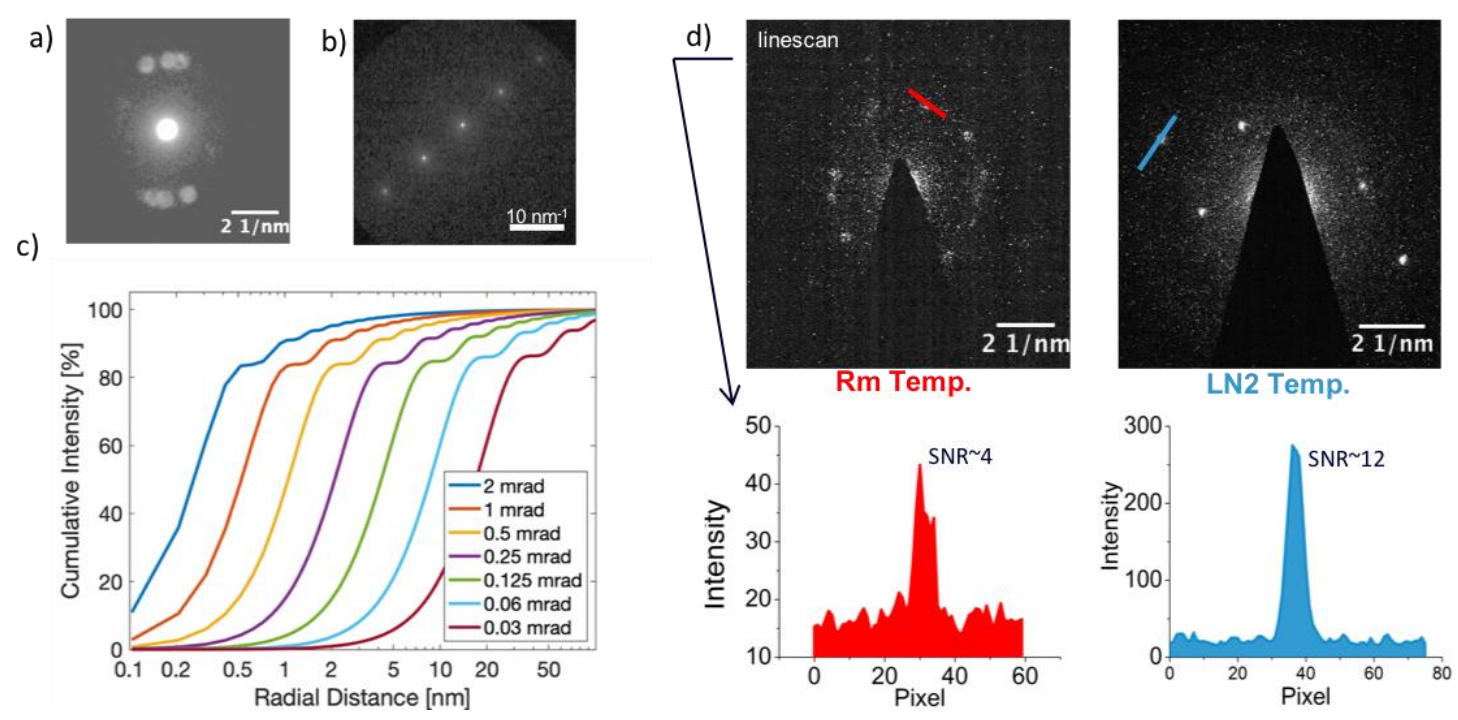

Figure 1. Typical diffraction patterns from polymer samples a) $40 \mu \mathrm{m}$ condenser aperture with $0.5 \mathrm{mrad}$ convergence angle; b) $2 \mu \mathrm{m}$ condenser aperture with $60 \mu \mathrm{rad}$ convergence angle; c) Theoretical dependence of real-space Airy-disk as a function of convergence angle; (d) Improvement of SNR at liquid N2 temperature of polyethylene.

\section{References}

[1] C Ophus, Microscopy and Microanalysis 25 (2019) p. 563.

[2] O Panova et al., Nature Materials 18 (2019) p. 860.

[3] M Gallagher-Jones et al., Communications Biology 2 (2019) 26.

[4] S Zeltmann et al., Ultramicroscopy 209 (2020) p. 112890.

[5] R Egerton et al., Micron 35 (2004) p. 399.

[6] Work at the Molecular Foundry was supported by the Office of Science, Office of Basic Energy Sciences, of the U.S. Department of Energy under Contract No. DE-AC02-05CH11231. MC was supported by the Electron Microscopy of Soft Matter Program from the Office of Science, Office of Basic Energy Sciences, Materials Sciences and Engineering Division of the U.S. Department of Energy under Contract No. DE-AC02-05CH11231. SEZ was supported by STROBE, a Science and Technology Center sponsored by the National Science Foundation. JD was supported by the Dow University Partnership Initiative Program. JC acknowledges additional support from the Presidential Early Career Award for Scientists and Engineers (PECASE) through the U.S. Department of Energy. 